\title{
A Note on Disk Counting in Toric Orbifolds
}

\author{
Kwokwai CHAN $\dagger^{1}$, Cheol-Hyun CHO ${ }^{\dagger^{2}}$, Siu-Cheong LAU $\dagger^{3}$, Naichung Conan LEUNG $\dagger^{\dagger^{4}}$ \\ and Hsian-Hua TSENG $\dagger^{5}$ \\ $\dagger^{1}$ Department of Mathematics, The Chinese University of Hong Kong, Shatin, Hong Kong \\ E-mail:kwchan@math.cuhk.edu.hk \\ $\dagger^{2}$ Department of Mathematical Sciences, Research Institute in Mathematics, \\ Seoul National University, Gwanak-Gu, Seoul, South Korea \\ E-mail: chocheol@snu.ac.kr \\ $\dagger^{3}$ Department of Mathematics and Statistics, Boston University, Boston, MA, USA \\ E-mail:lau@math.bu.edu \\ $\dagger^{4}$ The Institute of Mathematical Sciences and Department of Mathematics, \\ The Chinese University of Hong Kong, Shatin, Hong Kong \\ E-mail: leung@math.cuhk.edu.hk \\ $\dagger^{5}$ Department of Mathematics, Ohio State University, 100 Math Tower, \\ 231 West 18th Ave., Columbus, $\mathrm{OH}$ 43210, USA \\ E-mail: hhtseng@math.ohio-state.edu
}

Received January 24, 2020, in final form June 11, 2020; Published online June 17, 2020 https://doi.org/10.3842/SIGMA.2020.055

\begin{abstract}
We compute orbi-disk invariants of compact Gorenstein semi-Fano toric orbifolds by extending the method used for toric Calabi-Yau orbifolds. As a consequence the orbi-disc potential is analytic over complex numbers.
\end{abstract}

Key words: orbifold; toric; open Gromov-Witten invariants; mirror symmetry; SYZ

2020 Mathematics Subject Classification: 53D37; 14J33

\section{Introduction}

The mirror map plays a central role in the study of mirror symmetry. It provides a canonical local isomorphism between the Kähler moduli and the complex moduli of the mirror near a large complex structure limit. Such an isomorphism is crucial to counting of rational curves using mirror symmetry.

The mirror map is a transformation from the complex coordinates of the Hori-Vafa mirror moduli to the canonical coordinates obtained from period integrals. In [4] and [5], we derived an enumerative meaning of the inverse mirror maps for toric Calabi-Yau orbifolds and compact semi-Fano toric manifolds in terms of genus 0 open (orbifold) Gromov-Witten invariants (or (orbi-)disk invariants). Namely, we showed that coefficients of the inverse mirror map are equal to generating functions of virtual counts of stable (orbi-)disks bounded by a regular Lagrangian moment map fiber. In particular it gives a way to effectively compute all such invariants.

It is interesting to compare this with the mirror family constructed by Gross-Siebert [19], which is written in canonical coordinates [24]. In [19, Conjecture 0.2], it was conjectured that the wall-crossing functions in their construction are generating functions of open Gromov-Witten invariants. Our results verify this conjecture in the toric setting, namely, we showed that the SYZ mirror family [25], constructed using open Gromov-Witten invariants, is written in canonical coordinates. 
In this short note we extend our method in [4] to derive an explicit formula for the orbi-disk invariants in the case of compact Gorenstein semi-Fano toric orbifolds; see Theorem 3.6 for the explicit formulas. This proves [3, Conjecture] for such orbifolds, generalizing [5, Theorem 1.2]:

Theorem 1.1 (open mirror theorem). For a compact Gorenstein semi-Fano toric orbifold, the orbi-disk potential is equal to the (extended) Hori-Vafa superpotential via the mirror map.

See (3.6) for the definition of the orbi-disc potential. We remark that the open crepant resolution conjecture [3, Conjecture 1] may be studied using this computation and techniques of analytical continuation in [4, Appendix A].

Corollary 1.2. There exists an open neighborhood around the large volume limit where the orbi-disk potential converges.

This generalizes [5, Theorem 7.6] to the orbifold case.

\section{Preparation}

\section{$2.1 \quad$ Toric orbifolds}

\subsubsection{Construction}

Following [2], a stacky fan is the combinatorial data $\left(\Sigma, \boldsymbol{b}_{0}, \ldots, \boldsymbol{b}_{m-1}\right)$, where $\Sigma$ is a simplicial fan contained in the $\mathbb{R}$-vector space $N_{\mathbb{R}}:=N \otimes_{\mathbb{Z}} \mathbb{R}, N$ is a lattice of rank $n$, and $\left\{\boldsymbol{b}_{i} \mid 0 \leq i \leq\right.$ $m-1\} \subset N$ are generators of 1-dimensional cones of $\Sigma$. $\boldsymbol{b}_{i}$ are called the stacky vectors.

Choose $\boldsymbol{b}_{m}, \ldots, \boldsymbol{b}_{m^{\prime}-1} \in N$ so that they are contained in the support of the fan $\Sigma$ and they generate $N$ over $\mathbb{Z}$. An extended stacky fan in the sense of [22] is the data

$$
\left(\Sigma,\left\{\boldsymbol{b}_{i}\right\}_{i=0}^{m-1} \cup\left\{\boldsymbol{b}_{j}\right\}_{j=m}^{m^{\prime}-1}\right) .
$$

The vectors $\left\{\boldsymbol{b}_{j}\right\}_{j=m}^{m^{\prime}-1}$ are called extra vectors.

The fan map associated to an extended stacky fan (2.1) is defined by

$$
\phi: \quad \tilde{N}:=\bigoplus_{i=0}^{m^{\prime}-1} \mathbb{Z} e_{i} \rightarrow N, \quad \phi\left(e_{i}\right):=\boldsymbol{b}_{i} \quad \text { for } i=0, \ldots, m^{\prime}-1 .
$$

$\phi$ is surjective and yields an exact sequence of groups called the fan sequence:

$$
0 \longrightarrow \mathbb{L}:=\operatorname{Ker}(\phi) \stackrel{\psi}{\longrightarrow} \tilde{N} \stackrel{\phi}{\longrightarrow} N \longrightarrow 0 .
$$

Clearly $\mathbb{L} \simeq \mathbb{Z}^{m^{\prime}-n}$. Tensoring (2.2) with $\mathbb{C}^{\times}$yields the following sequence:

$$
0 \longrightarrow G:=\mathbb{L} \otimes_{\mathbb{Z}} \mathbb{C}^{\times} \longrightarrow \widetilde{N} \otimes_{\mathbb{Z}} \mathbb{C}^{\times} \simeq\left(\mathbb{C}^{\times}\right)^{m^{\prime}} \stackrel{\phi_{\mathbb{C}^{\times}}}{\longrightarrow} \mathbb{T}:=N \otimes_{\mathbb{Z}} \mathbb{C}^{\times} \rightarrow 0,
$$

which is exact. Note that $G$ is an algebraic torus.

By definition, the set of anti-cones is

$$
\mathcal{A}:=\left\{I \subset\left\{0,1, \ldots, m^{\prime}-1\right\} \mid \sum_{i \notin I} \mathbb{R}_{\geq 0} \boldsymbol{b}_{i} \text { is a cone in } \Sigma\right\} .
$$

This terminology is justified because for $I \in \mathcal{A}$, the complement of $I$ in $\left\{0,1, \ldots, m^{\prime}-1\right\}$ indexes generators of a cone in $\Sigma$. For $I \in \mathcal{A}$, the collection $\left\{Z_{i} \mid i \in I\right\}$ generates an ideal in $\mathbb{C}\left[Z_{0}, \ldots, Z_{m^{\prime}-1}\right]$, which in turn determines a subvariety $\mathbb{C}^{I} \subset \mathbb{C}^{m^{\prime}}$. Set

$$
U_{\mathcal{A}}:=\mathbb{C}^{m^{\prime}} \backslash \bigcup_{I \notin \mathcal{A}} \mathbb{C}^{I}
$$


The map $G \rightarrow\left(\mathbb{C}^{\times}\right)^{m^{\prime}}$ in $(2.3)$ defines a $G$-action on $\mathbb{C}^{m^{\prime}}$ and hence a $G$-action on $U_{\mathcal{A}}$. This action is effective and has finite stabilizers, because $N$ is torsion-free (see [22, Section 2]). The toric orbifold associated to $\left(\Sigma,\left\{\boldsymbol{b}_{i}\right\}_{i=0}^{m-1} \cup\left\{\boldsymbol{b}_{j}\right\}_{j=m}^{m^{\prime}-1}\right)$ is defined to be the following quotient stack:

$$
\mathcal{X}_{\Sigma}:=\left[U_{\mathcal{A}} / G\right]
$$

The standard $\left(\mathbb{C}^{\times}\right)^{m^{\prime}}$-action on $U_{\mathcal{A}}$ induces a $\mathbb{T}$-action on $\mathcal{X}_{\Sigma}$ via $(2.3)$.

The coarse moduli space of the toric orbifold $\mathcal{X}_{\Sigma}$ is the toric variety $X_{\Sigma}$ associated to the fan $\Sigma$. In this paper we assume that $X_{\Sigma}$ is semi-projective, i.e., $X_{\Sigma}$ has a $\mathbb{T}$-fixed point and the natural map $X_{\Sigma} \rightarrow \operatorname{Spec} H^{0}\left(X_{\Sigma}, \mathcal{O}_{X_{\Sigma}}\right)$ is projective, or equivalently, $X_{\Sigma}$ arises as a GIT quotient of a complex vector space by an abelian group (see [20, Section 2]). This assumption is required for the toric mirror theorem of [11] to hold. More detailed discussions on semi-projective toric varieties can be found in [12, Section 7.2].

\subsubsection{Twisted sectors}

Consider a $d$-dimensional cone $\sigma \in \Sigma$ generated by $\boldsymbol{b}_{\sigma}=\left(\boldsymbol{b}_{i_{1}}, \ldots, \boldsymbol{b}_{i_{d}}\right)$. Define

$$
\operatorname{Box}_{\boldsymbol{b}_{\sigma}}:=\left\{\nu \in N \mid \nu=\sum_{k=1}^{d} t_{k} \boldsymbol{b}_{i_{k}}, t_{k} \in[0,1) \cap \mathbb{Q}\right\} \text {. }
$$

$\left\{\boldsymbol{b}_{i_{1}}, \ldots, \boldsymbol{b}_{i_{d}}\right\}$ generates a submodule $N_{\boldsymbol{b}_{\sigma}} \subset N$. One can check that there is a bijection between Box $_{\boldsymbol{b}_{\sigma}}$ and the finite group $G_{\boldsymbol{b}_{\sigma}}:=\left(N \cap \operatorname{Span}_{\mathbb{R}} \boldsymbol{b}_{\sigma}\right) / N_{\boldsymbol{b}_{\sigma}}$. Furthermore, if $\tau$ is a subcone of $\sigma$, then $\operatorname{Box}_{\boldsymbol{b}_{\tau}} \subset \operatorname{Box}_{\boldsymbol{b}_{\sigma}}$. Define

$$
\begin{aligned}
& \operatorname{Box}_{\boldsymbol{b}_{\sigma}}^{\circ}: \operatorname{Box}_{\boldsymbol{b}_{\sigma}} \backslash \bigcup_{\tau \supsetneqq \sigma} \operatorname{Box}_{\boldsymbol{b}_{\tau}}, \quad \operatorname{Box}(\Sigma):=\bigcup_{\sigma \in \Sigma^{(n)}} \operatorname{Box}_{\boldsymbol{b}_{\sigma}}=\bigsqcup_{\sigma \in \Sigma} \operatorname{Box}_{\boldsymbol{b}_{\sigma}}^{\circ}, \\
& \operatorname{Box}^{\prime}(\Sigma)=\operatorname{Box}(\Sigma) \backslash\{0\},
\end{aligned}
$$

where $\Sigma^{(n)}$ is the set of $n$-dimensional cones in $\Sigma$.

Following the description of the inertia orbifold of $\mathcal{X}_{\Sigma}$ in [2], for $\nu \in \operatorname{Box}(\Sigma)$, we denote by $\mathcal{X}_{\nu}$ the corresponding component of the inertia orbifold of $\mathcal{X}:=\mathcal{X}_{\Sigma}$. Note that $\mathcal{X}_{0}=\mathcal{X}_{\Sigma}$ as orbifolds. Elements $\nu \in \operatorname{Box}^{\prime}(\Sigma)$ correspond to twisted sectors of $\mathcal{X}$, namely non-trivial connected components of the inertia orbifold of $\mathcal{X}$.

Following [7], the direct sum of singular cohomology groups of components of the inertia orbifold of $\mathcal{X}$, subject to a degree shift, is called the Chen-Ruan orbifold cohomology $H_{\mathrm{CR}}^{*}(\mathcal{X} ; \mathbb{Q})$ of $\mathcal{X}$. More precisely,

$$
H_{\mathrm{CR}}^{d}(\mathcal{X} ; \mathbb{Q})=\bigoplus_{\nu \in \operatorname{Box}} H^{d-2 \operatorname{age}(\nu)}\left(\mathcal{X}_{\nu} ; \mathbb{Q}\right)
$$

where age $(\nu)$ is called the degree shifting number ${ }^{1}$ in [7] of the twisted sector $\mathcal{X}_{\nu}$. In case of toric orbifolds, age has a combinatorial description [2]: if $\nu=\sum_{k=1}^{d} t_{k} \boldsymbol{b}_{i_{k}} \in \operatorname{Box}(\Sigma)$ where $\left\{\boldsymbol{b}_{i_{1}}, \ldots, \boldsymbol{b}_{i_{d}}\right\}$ generates a cone in $\Sigma$, then

$$
\operatorname{age}(\nu)=\sum_{k=1}^{d} t_{k} \in \mathbb{Q} \geq 0
$$

\footnotetext{
${ }^{1}$ Following Miles Reid, it is now more commonly called age.
} 
Using $\mathbb{T}$-actions on twisted sectors induced from that on $\mathcal{X}$, we can define $\mathbb{T}$-equivariant ChenRuan orbifold cohomology $H_{\mathrm{CR}, \mathbb{T}}^{*}(\mathcal{X} ; \mathbb{Q})$ by replacing singular cohomology with $\mathbb{T}$-equivariant cohomology $H_{\mathbb{T}}^{*}(-)$. Namely

$$
H_{\mathrm{CR}, \mathbb{T}}^{d}(\mathcal{X} ; \mathbb{Q})=\bigoplus_{\nu \in \operatorname{Box}} H_{\mathbb{T}}^{d-2 \operatorname{age}(\nu)}\left(\mathcal{X}_{\nu} ; \mathbb{Q}\right)
$$

By general properties of equivariant cohomology, $H_{\mathrm{CR}, \mathbb{T}}^{*}(\mathcal{X} ; \mathbb{Q})$ is a module over $H_{\mathbb{T}}^{*}(\mathrm{pt}, \mathbb{Q})$ and admits a map $H_{\mathrm{CR}, \mathbb{T}}^{*}(\mathcal{X} ; \mathbb{Q}) \rightarrow H_{\mathrm{CR}}^{*}(\mathcal{X} ; \mathbb{Q})$ called non-equivariant limit.

\subsubsection{Toric divisors, Kähler cones, and Mori cones}

We continue using the notations in Sections 2.1.1 and 2.1.2. Applying $\operatorname{Hom}_{\mathbb{Z}}(-, \mathbb{Z})$ to the fan sequence $(2.2)$, we obtain the following exact sequence: ${ }^{2}$

$$
0 \longrightarrow M:=N^{\vee}=\operatorname{Hom}(N, \mathbb{Z}) \stackrel{\phi^{\vee}}{\longrightarrow} \widetilde{M}:=\widetilde{N}^{\vee}=\operatorname{Hom}(\widetilde{N}, \mathbb{Z}) \stackrel{\psi^{\vee}}{\longrightarrow} \mathbb{L}^{\vee}=\operatorname{Hom}(\mathbb{L}, \mathbb{Z}) \longrightarrow 0,
$$

which is called the divisor sequence. Line bundles on $\mathcal{X}=\left[\mathcal{U}_{\mathcal{A}} / G\right]$ correspond to $G$-equivariant line bundles on $\mathcal{U}_{\mathcal{A}}$. In view of $(2.3), \mathbb{T}$-equivariant line bundles on $\mathcal{X}$ correspond to $\left(\mathbb{C}^{\times}\right)^{m^{\prime}}$ equivariant line bundles on $\mathcal{U}_{\mathcal{A}}$. Because the codimension of $\cup_{I \notin \mathcal{A}} \mathbb{C}^{I} \subset \mathbb{C}^{m^{\prime}}$ is at least 2 , the Picard groups satisfy:

$$
\operatorname{Pic}(\mathcal{X}) \simeq \operatorname{Hom}\left(G, \mathbb{C}^{\times}\right) \simeq \mathbb{L}^{\vee}, \quad \operatorname{Pic}_{\mathbb{T}}(\mathcal{X}) \simeq \operatorname{Hom}\left(\left(\mathbb{C}^{\times}\right)^{m^{\prime}}, \mathbb{C}^{\times}\right) \simeq \widetilde{N}^{\vee}=\widetilde{M}
$$

The natural map $\operatorname{Pic}_{\mathbb{T}}(\mathcal{X}) \rightarrow \operatorname{Pic}(\mathcal{X})$ is identified with the map $\psi^{\vee}: \widetilde{M} \rightarrow \mathbb{L}^{\vee}$ appearing in the divisor sequence.

The elements $\left\{e_{i}^{\vee} \mid i=0,1, \ldots, m^{\prime}-1\right\} \subset \widetilde{M} \simeq \operatorname{Pic}_{\mathbb{T}}(\mathcal{X})$ dual to $\left\{e_{i} \mid i=0,1, \ldots, m^{\prime}-1\right\} \subset \tilde{N}$ correspond to $\mathbb{T}$-equivariant line bundle on $\mathcal{X}$ which we denote by $D_{i}^{\mathbb{T}}, i=0,1, \ldots, m^{\prime}-1$. The collection

$$
\left\{D_{i}:=\psi^{\vee}\left(e_{i}^{\vee}\right) \mid 0 \leq i \leq m-1\right\} \subset \mathbb{L}^{\vee} \simeq \operatorname{Pic}(\mathcal{X})
$$

consists of toric prime divisors corresponding to the generators $\left\{\boldsymbol{b}_{i} \mid 0 \leq i \leq m-1\right\}$ of 1-dimensional cones in $\Sigma$. Elements $D_{i}^{\mathbb{T}}, 0 \leq i \leq m-1$ are $\mathbb{T}$-equivariant lifts of these divisors. There are natural maps

$$
\begin{aligned}
& \widetilde{M} \otimes \mathbb{Q}^{\psi^{\vee}} \stackrel{\otimes \mathbb{Q}}{\rightarrow} \mathbb{L}^{\vee} \otimes \mathbb{Q}, \\
& (\widetilde{M} \otimes \mathbb{Q}) /\left(\sum_{j=m}^{m^{\prime}-1} \mathbb{Q} D_{j}^{\mathbb{T}}\right) \simeq H_{\mathbb{T}}^{2}(\mathcal{X}, \mathbb{Q}) \rightarrow H^{2}(\mathcal{X}, \mathbb{Q}) \simeq\left(\mathbb{L}^{\vee} \otimes \mathbb{Q}\right) /\left(\sum_{j=m}^{m^{\prime}-1} \mathbb{Q} D_{j}\right) .
\end{aligned}
$$

Together with the natural quotient maps, they fit into a commutative diagram.

As explained in $\left[21\right.$, Section 3.1.2], there is a canonical splitting of the quotient map $\mathbb{L}^{\vee} \otimes \mathbb{Q} \rightarrow$ $H^{2}(\mathcal{X} ; \mathbb{Q})$. For $m \leq j \leq m^{\prime}-1$, let $I_{j} \in \mathcal{A}$ be the anticone of the cone containing $\boldsymbol{b}_{j}$. This allows us to write $\boldsymbol{b}_{j}=\sum_{i \notin I_{j}} c_{j i} \boldsymbol{b}_{i}$ for $c_{j i} \in \mathbb{Q}_{\geq 0}$.

Tensoring the fan sequence $(2.2)$ with $\mathbb{Q}$, we may find a unique $D_{j}^{\vee} \in \mathbb{L} \otimes \mathbb{Q}$ such that values of the natural pairing $\langle-,-\rangle$ between $\mathbb{L}^{\vee}$ and $\mathbb{L}$ satisfy

$$
\left\langle D_{i}, D_{j}^{\vee}\right\rangle= \begin{cases}1 & \text { if } i=j, \\ -c_{j i} & \text { if } i \notin I_{j}, \\ 0 & \text { if } i \in I_{j} \backslash\{j\} .\end{cases}
$$

\footnotetext{
${ }^{2}$ The map $\psi^{\vee}: \widetilde{M} \rightarrow \mathbb{L}^{\vee}$ is surjective since $N$ is torsion-free.
} 
Using $D_{j}^{\vee}$ we get a decomposition

$$
\mathbb{L}^{\vee} \otimes \mathbb{Q}=\operatorname{Ker}\left(\left(D_{m}^{\vee}, \ldots, D_{m^{\prime}-1}^{\vee}\right): \mathbb{L}^{\vee} \otimes \mathbb{Q} \rightarrow \mathbb{Q}^{m^{\prime}-m}\right) \oplus \bigoplus_{j=m}^{m^{\prime}-1} \mathbb{Q} D_{j} .
$$

We can view $H^{2}(\mathcal{X} ; \mathbb{Q})$ as a subspace of $\mathbb{L}^{\vee} \otimes \mathbb{Q}$ because $\operatorname{Ker}\left(\left(D_{m}^{\vee}, \ldots, D_{m^{\prime}-1}^{\vee}\right)\right)$ can be identified with $H^{2}(\mathcal{X} ; \mathbb{Q})$ via the map $\mathbb{L}^{\vee} \otimes \mathbb{Q} \rightarrow H^{2}(\mathcal{X} ; \mathbb{Q})$.

Define extended Kähler cone of $\mathcal{X}$ to be

$$
\widetilde{C}_{\mathcal{X}}:=\bigcap_{I \in \mathcal{A}}\left(\sum_{i \in I} \mathbb{R}_{>0} D_{i}\right) \subset \mathbb{L}^{\vee} \otimes \mathbb{R}
$$

The Kähler cone $C_{\mathcal{X}}$ is the image of $\widetilde{C}_{\mathcal{X}}$ under $\mathbb{L}^{\vee} \otimes \mathbb{R} \rightarrow H^{2}(\mathcal{X} ; \mathbb{R})$. The splitting (2.5) of $\mathbb{L}^{\vee} \otimes \mathbb{Q}$ yields a splitting $\widetilde{C}_{\mathcal{X}}=C_{\mathcal{X}}+\sum_{j=m}^{m^{\prime}-1} \mathbb{R}_{>0} D_{j}$

By $(2.2), \mathbb{L}^{\vee}$ has rank equal to $r:=m^{\prime}-n$. The rank of $H_{2}(\mathcal{X} ; \mathbb{Z})$ is $r^{\prime}:=r-\left(m^{\prime}-m\right)=m-n$. We choose an integral basis

$$
\left\{p_{1}, \ldots, p_{r}\right\} \subset \mathbb{L}^{\vee}
$$

such that $p_{a}$ is in the closure of $\widetilde{C}_{\mathcal{X}}$ for all $a$ and $p_{r^{\prime}+1}, \ldots, p_{r} \in \sum_{i=m}^{m^{\prime}-1} \mathbb{R}_{\geq 0} D_{i}$. We get a nef basis $\left\{\bar{p}_{1}, \ldots, \bar{p}_{r^{\prime}}\right\}$ for $H^{2}(\mathcal{X} ; \mathbb{Q})$ as images of $\left\{p_{1}, \ldots, p_{r^{\prime}}\right\}$ under the quotient map $\mathbb{L}^{\vee} \otimes \mathbb{Q} \rightarrow H^{2}(\mathcal{X} ; \mathbb{Q})$. For $r^{\prime}+1 \leq a \leq r$, the images satisfies $\bar{p}_{a}=0$.

We choose equivariant lifts of $p_{a}$ 's, namely $\left\{p_{1}^{\mathbb{T}}, \ldots, p_{r}^{\mathbb{T}}\right\} \subset \widetilde{M} \otimes \mathbb{Q}$ such that $\psi^{\vee}\left(p_{a}^{\mathbb{T}}\right)=p_{a}$ for all $a$. We also require that for $a=r^{\prime}+1, \ldots, r$ the images $\bar{p}_{a}^{\mathbb{T}}$ of $p_{a}^{\mathbb{T}}$ under the natural map $\widetilde{M} \otimes \mathbb{Q} \rightarrow H_{\mathbb{T}}^{2}(\mathcal{X}, \mathbb{Q})$ satisfies $\bar{p}_{a}^{\mathbb{T}}=0$.

The coefficients $Q_{i a} \in \mathbb{Z}$ in the equations $D_{i}=\sum_{a=1}^{r} Q_{i a} p_{a}$ assemble to a matrix $\left(Q_{i a}\right)$. The images $^{3} \bar{D}_{i}$ of $D_{i}$ under the map $\mathbb{L}^{\vee} \otimes \mathbb{Q} \rightarrow H^{2}(\mathcal{X} ; \mathbb{Q})$ can be expressed as

$$
\bar{D}_{i}=\sum_{a=1}^{r^{\prime}} Q_{i a} \bar{p}_{a}, \quad i=0, \ldots, m-1
$$

Their equivariant lifts $\bar{D}_{i}^{\mathbb{T}}$ can be expressed as

$$
\bar{D}_{i}^{\mathbb{T}}=\sum_{a=1}^{r^{\prime}} Q_{i a} \bar{p}_{a}^{\mathbb{T}}+\lambda_{i}, \quad \text { where } \quad \lambda_{i} \in H^{2}(B \mathbb{T} ; \mathbb{Q}) .
$$

For $i=m, \ldots, m^{\prime}-1$, we have $\bar{D}_{i}=0$ in $H^{2}(\mathcal{X} ; \mathbb{R})$ and $\bar{D}_{i}^{\mathbb{T}}=0$.

Localization gives the following description of $H_{\mathrm{CR}, \mathbb{T}}^{\leq 2}$ :

$$
H_{\mathrm{CR}, \mathbb{T}}^{0}\left(\mathcal{X}, K_{\mathbb{T}}\right)=K_{\mathbb{T}} \mathbf{1}, \quad H_{\mathrm{CR}, \mathbb{T}}^{2}\left(\mathcal{X}, K_{\mathbb{T}}\right)=\bigoplus_{a=1}^{r^{\prime}} K_{\mathbb{T}} \bar{p}_{a}^{\mathbb{T}} \oplus \bigoplus_{\nu \in \operatorname{Box}, \operatorname{age}(\nu)=1} K_{\mathbb{T}} \mathbf{1}_{\nu}
$$

Here $K_{\mathbb{T}}$ is the field of fractions of $H_{\mathbb{T}}^{*}(\mathrm{pt}, \mathbb{Q}), \mathbf{1} \in H^{0}(\mathcal{X}, \mathbb{Q})$ and $\mathbf{1}_{\nu} \in H^{0}\left(\mathcal{X}_{\nu}, \mathbb{Q}\right)$ are fundamental classes.

\footnotetext{
${ }^{3} \bar{D}_{i}$ is the class of the toric prime divisor $D_{i}$.
} 
Let

$$
\left\{\gamma_{1}, \ldots, \gamma_{r}\right\} \subset \mathbb{L}, \quad \gamma_{a}=\sum_{i=0}^{m^{\prime}-1} Q_{i a} e_{i} \in \tilde{N},
$$

be the basis dual to $\left\{p_{1}, \ldots, p_{r}\right\} \subset \mathbb{L}^{\vee} . H_{2}^{\text {eff }}(\mathcal{X} ; \mathbb{Q})$ admits a basis $\left\{\gamma_{1}, \ldots, \gamma_{r^{\prime}}\right\}$, and we have $Q_{i a}=0$ when $m \leq i \leq m^{\prime}-1$ and $1 \leq a \leq r^{\prime}$.

Set

$$
\begin{aligned}
& \mathbb{K}:=\left\{d \in \mathbb{L} \otimes \mathbb{Q} \mid\left\{j \in\left\{0,1, \ldots, m^{\prime}-1\right\} \mid\left\langle D_{j}, d\right\rangle \in \mathbb{Z}\right\} \in \mathcal{A}\right\}, \\
& \mathbb{K}_{\mathrm{eff}}:=\left\{d \in \mathbb{L} \otimes \mathbb{Q} \mid\left\{j \in\left\{0,1, \ldots, m^{\prime}-1\right\} \mid\left\langle D_{j}, d\right\rangle \in \mathbb{Z}_{\geq 0}\right\} \in \mathcal{A}\right\} .
\end{aligned}
$$

Elements of $\mathbb{K}_{\mathrm{eff}}$ should be interpreted as effective curve classes. Elements of $\mathbb{K}_{\mathrm{eff}} \cap H_{2}(\mathcal{X} ; \mathbb{R})$ should be viewed as classes of stable maps $\mathbb{P}(1, m) \rightarrow \mathcal{X}$ for some $m \in \mathbb{Z}_{\geq 0}$. See, e.g., [21, Section 3.1] for more details.

Definition 2.1. A toric orbifold $\mathcal{X}$ is called semi-Fano if $c_{1}(\mathcal{X}) \cdot \alpha>0$ for every effective curve class $\alpha$, in other words, $-K_{\mathcal{X}}$ is nef.

For $d \in \mathbb{K}$, put $^{4}$

$$
\nu(d):=\sum_{i=0}^{m^{\prime}-1}\left\lceil\left\langle D_{i}, d\right\rangle\right\rceil \boldsymbol{b}_{i} \in N
$$

and let $I_{d}:=\left\{j \in\left\{0,1, \ldots, m^{\prime}-1\right\} \mid\left\langle D_{j}, d\right\rangle \in \mathbb{Z}\right\} \in \mathcal{A}$. Then $\nu(d) \in$ Box because

$$
\nu(d)=\sum_{i=0}^{m^{\prime}-1}\left(\left\{-\left\langle D_{i}, d\right\rangle\right\}+\left\langle D_{i}, d\right\rangle\right) \boldsymbol{b}_{i}=\sum_{i=0}^{m^{\prime}-1}\left\{-\left\langle D_{i}, d\right\rangle\right\} \boldsymbol{b}_{i}=\sum_{i \notin I_{d}}\left\{-\left\langle D_{i}, d\right\rangle\right\} \boldsymbol{b}_{i} .
$$

\subsection{Genus 0 open orbifold GW invariants according to [9]}

Let $(\mathcal{X}, \omega)$ be a toric Kähler orbifold of complex dimension $n$, equipped with the standard toric complex structure $J_{0}$ and a toric Kähler structure $\omega$. Denote by $(\Sigma, \boldsymbol{b})$ the stacky fan that defines $\mathcal{X}$, where $\boldsymbol{b}=\left(\boldsymbol{b}_{0}, \ldots, \boldsymbol{b}_{m-1}\right)$ and $\boldsymbol{b}_{i}=c_{i} v_{i}$.

Let $L \subset \mathcal{X}$ be a Lagrangian torus fiber of the moment map $\mu_{0}: \mathcal{X} \rightarrow M_{\mathbb{R}}:=M \otimes_{\mathbb{Z}} \mathbb{R}$, and let $\beta \in \pi_{2}(\mathcal{X}, L)=H_{2}(\mathcal{X}, L ; \mathbb{Z})$ be a relative homotopy class.

\subsubsection{Holomorphic orbi-disks and their moduli spaces}

A holomorphic orbi-disk in $\mathcal{X}$ with boundary in $L$ is a continuous map

$$
w:(\mathbb{D}, \partial \mathbb{D}) \rightarrow(\mathcal{X}, L)
$$

satisfying the following conditions:

1. $\left(\mathbb{D}, z_{1}^{+}, \ldots, z_{l}^{+}\right)$is an orbi-disk with interior marked points $z_{1}^{+}, \ldots, z_{l}^{+}$. More precisely $\mathbb{D}$ is analytically the disk $D^{2} \subset \mathbb{C}$ so that for $j=1, \ldots, l$, the orbifold structure at $z_{j}^{+}$is given by a disk neighborhood of $z_{j}^{+}$uniformized by the branched covering map br: $z \rightarrow z^{m_{j}}$ for some $m_{j} \in \mathbb{Z}_{>0}$. (If $m_{j}=1, z_{j}^{+}$is not an orbifold point.)

\footnotetext{
${ }^{4}$ For a real number $\lambda \in \mathbb{R}$, let $\lceil\lambda\rceil,\lfloor\lambda\rfloor$ and $\{\lambda\}$ denote the ceiling, floor and fractional part of $\lambda$ respectively.
} 
2. For any $z_{0} \in \mathbb{D}$, there is a disk neighborhood of $z_{0}$ with a branched covering map br: $z \rightarrow$ $z^{m}$, and there is a local chart $\left(V_{w\left(z_{0}\right)}, G_{w\left(z_{0}\right)}, \pi_{w\left(z_{0}\right)}\right)$ of $\mathcal{X}$ at $w\left(z_{0}\right)$ and a local holomorphic lifting $\widetilde{w}_{z_{0}}$ of $w$ satisfying $w \circ \mathrm{br}=\pi_{w\left(z_{0}\right)} \circ \widetilde{w}_{z_{0}}$.

3. The map $w$ is good (in the sense of Chen-Ruan [6]) and representable. In particular, for each $z_{j}^{+}$, the associated group homomorphism

$$
h_{p}: \mathbb{Z}_{m_{j}} \rightarrow G_{w\left(z_{j}^{+}\right)}
$$

between local groups which makes $\widetilde{w}_{z_{j}^{+}}$equivariant, is injective.

The type of a map $w$ as above is defined to be $\boldsymbol{x}:=\left(\mathcal{X}_{\nu_{1}}, \ldots, \mathcal{X}_{\nu_{l}}\right)$. Here $\nu_{j} \in \operatorname{Box}(\Sigma)$ is the image of the generator $1 \in \mathbb{Z}_{m_{j}}$ under $h_{j}$.

There are two notions of Maslov index for an orbi-disk. The desingularized Maslov index $\mu^{\mathrm{de}}$ is defined by desingularizing the interior singularities of the pull-back bundle $w^{*} T \mathcal{X}$. Namely, the bundle $w^{*} T \mathcal{X}$ over an orbi-disk $\left(\mathbb{D}, z_{1}^{+}, \ldots, z_{l}^{+}\right)$cannot be trivialized due to the orbifold structure, but we can obtain another bundle $\left|w^{*} T \mathcal{X}\right|$ by modifying the bundle near orbifold points (see Chen-Ruan [6] for more details). This is called a desingularization of $w^{*} T \mathcal{X}$ and it is a smooth bundle over the orbi-disk, hence is a trivial bundle. We can compute the Maslov index of the boundary Lagrangian loop relative to this trivialization, and it is called the desingularized Maslov index. See [9, Section 3] for more details and [9, Section 5] for an explicit formula in the toric case.

The Chern-Weil $(C W)$ Maslov index $\mu_{\mathrm{CW}}$ is defined as the integral of the curvature of a unitary connection on $w^{*} T \mathcal{X}$ which preserves the Lagrangian boundary condition, see [10] (and also [9, Section 3.3] for a relation with $\mu^{\mathrm{de}}$ ). The following lemma, which appeared as [4, Lemma 3.1], computes the CW Maslov indices of disks. This is an orbifold version of the formula in [1, Lemma 3.1].

Lemma 2.2. Let $(\mathcal{X}, \omega, J)$ be a Kähler orbifold of complex dimension $n$. Let $\Omega$ be a non-zero meromorphic $n$-form on $\mathcal{X}$ which has at worst simple poles. Let $D \subset \mathcal{X}$ be the pole divisor of $\Omega$. Suppose also that the generic points of $D$ are smooth. Then for a special Lagrangian submanifold $L \subset \mathcal{X} \backslash D$, the $C W$ Maslov index of a class $\beta \in \pi_{2}(\mathcal{X}, L)$ is given by

$$
\mu_{\mathrm{CW}}(\beta)=2 \beta \cdot D \text {. }
$$

Here, $\beta \cdot D$ is defined by writing $\beta$ as a fractional linear combination of homotopy classes of smooth disks.

The classification of orbi-disks in a symplectic toric orbifold has been worked out in $[9$, Theorem 6.2]. It is similar to the classification of holomorphic discs in toric manifolds [8]. In the classification, the basic disks corresponding to the stacky vectors (and twisted sectors) play a basic role.

Theorem 2.3 ([9, Corollaries 6.3 and 6.4]). Let $\mathcal{X}$ be a toric Kähler orbifold and let $L$ be a fiber of the toric moment map.

1. The smooth holomorphic disks of Maslov index 2 (modulo $\mathbb{T}^{n}$-action and reparametrizations of the domain) are in bijective correspondence with the stacky vectors $\left\{\boldsymbol{b}_{0}, \ldots, \boldsymbol{b}_{m-1}\right\}$. Denote the homotopy classes of these disks by $\beta_{0}, \ldots, \beta_{m-1}$.

2. The holomorphic orbi-disks with one interior orbifold marked point and desingularized Maslov index 0 (modulo $\mathbb{T}^{n}$-action and reparametrizations of the domain) are in bijective correspondence with the twisted sectors $\nu \in \operatorname{Box}^{\prime}(\Sigma)$ of the toric orbifold $\mathcal{X}$. Denote the homotopy classes of these orbi-disks by $\beta_{\nu}$. 
Lemma 2.4 ([9, Lemma 9.1]). For $\mathcal{X}$ and $L$ as above, the relative homotopy group $\pi_{2}(\mathcal{X}, L)$ is generated by the classes $\beta_{i}$ for $i=0, \ldots, m-1$ together with $\beta_{\nu}$ for $\nu \in \operatorname{Box}^{\prime}(\Sigma)$.

As in [9], these generators of $\pi_{2}(\mathcal{X}, L)$ are called basic disk classes. They are the analogue of Maslov index 2 disk classes in toric manifolds.

Let

$$
\mathcal{M}_{k+1, l}^{\text {op,main }}(\mathcal{X}, L, \beta, \boldsymbol{x})
$$

be the moduli space of good representable stable maps from bordered orbifold Riemann surfaces of genus zero with $k+1$ boundary marked points $z_{0}, z_{1}, \ldots, z_{k}$ and $l$ interior (orbifold) marked points $z_{1}^{+}, \ldots, z_{l}^{+}$in the homotopy class $\beta$ of type $\boldsymbol{x}=\left(\mathcal{X}_{\nu_{1}}, \ldots, \mathcal{X}_{\nu_{l}}\right)$. The superscript "main" is meant to indicate the connected component on which the boundary marked points respect the cyclic order of $S^{1}=\partial D^{2}$. According to $\left[9\right.$, Lemma 2.5], $\mathcal{M}_{k+1, l}^{\text {op,main }}(\mathcal{X}, L, \beta, \boldsymbol{x})$ has real virtual dimension

$$
n+\mu_{\mathrm{CW}}(\beta)+k+1+2 l-3-2 \sum_{j=1}^{l} \operatorname{age}\left(\nu_{j}\right) .
$$

By [9, Proposition 9.4], if $\mathcal{M}_{1,1}^{\text {op,main }}(\mathcal{X}, L, \beta)$ is non-empty and if $\partial \beta$ is not in the sublattice generated by $\boldsymbol{b}_{0}, \ldots, \boldsymbol{b}_{m-1}$, then there exist $\nu \in \operatorname{Box}^{\prime}(\Sigma), k_{0}, \ldots, k_{m-1} \in \mathbb{N}$ and $\alpha \in H_{2}^{\text {eff }}(\mathcal{X})$ such that $\beta=\beta_{\nu}+\sum_{i=0}^{m-1} k_{i} \beta_{i}+\alpha$, where $\alpha$ is realized by a union of holomorphic (orbi-)spheres. The $\mathrm{CW}$ Maslov index of $\beta$ written in this way is given by $\mu_{\mathrm{CW}}(\beta)=2 \operatorname{age}(\nu)+2 \sum_{i=0}^{m-1} k_{i}+2 c_{1}(\mathcal{X}) \cdot \alpha$.

\subsubsection{Orbi-disk invariants}

Pick twisted sectors $\mathcal{X}_{\nu_{1}}, \ldots, \mathcal{X}_{\nu_{l}}$ of the toric orbifold $\mathcal{X}$. Consider the moduli space

$$
\mathcal{M}_{1, l}^{\text {op,main }}(\mathcal{X}, L, \beta, \boldsymbol{x})
$$

of good representable stable maps from bordered orbifold Riemann surfaces of genus zero with one boundary marked point and $l$ interior orbifold marked points of type $\boldsymbol{x}=\left(\mathcal{X}_{\nu_{1}}, \ldots, \mathcal{X}_{\nu_{l}}\right)$ representing the class $\beta \in \pi_{2}(\mathcal{X}, L)$. According to [9], $\mathcal{M}_{1, l}^{\text {op,main }}(\mathcal{X}, L, \beta, \boldsymbol{x})$ can be equipped with a virtual fundamental chain, which has an expected dimension $n$ if the following equality holds:

$$
\mu_{\mathrm{CW}}(\beta)=2+\sum_{j=1}^{l}\left(2 \cdot \operatorname{age}\left(\nu_{j}\right)-2\right) .
$$

Throughout the paper, we make the following assumptions.

Assumption 2.5. We assume that the toric orbifold $\mathcal{X}$ is semi-Fano (see Definition 2.1) and Gorenstein. ${ }^{5}$ Moreover, we assume that the type $\boldsymbol{x}$ consists of twisted sectors with age $\leq 1 .^{6}$

Then the age of every twisted sector of $\mathcal{X}$ is a non-negative integer. Since a basic orbi-disk class $\beta_{\nu}$ has Maslov index 2 age $(\nu)$, we see that every non-constant stable disk class has at least Maslov index 2 .

\footnotetext{
${ }^{5}$ This means that $K_{\mathcal{X}}$ is Cartier.

${ }^{6}$ This assumption does not impose any restriction in the construction of the SYZ mirror over $H_{\mathrm{CR}}^{\leq 2}(\mathcal{X})$. We do not discuss mirror construction in this paper.
} 
Moreover, the virtual fundamental chain $\left[\mathcal{M}_{1, l}^{\text {op,main }}(\mathcal{X}, L, \beta, \boldsymbol{x})\right]^{\text {vir }}$ has expected dimension $n$ when $\mu_{\mathrm{CW}}(\beta)=2$, and in fact we get a virtual fundamental cycle because $\beta$ attains the minimal Maslov index, thus preventing disk bubbling to occur. Therefore the following definition of genus 0 open orbifold $G W$ invariants (also known as orbi-disk invariants) is independent of the choice of perturbations of the Kuranishi structures: ${ }^{7}$

Definition 2.6 (orbi-disk invariants). Let $\beta \in \pi_{2}(\mathcal{X}, L)$ be a relative homotopy class with Maslov index given by (2.6). Suppose that the moduli space $\mathcal{M}_{1, l}^{\text {op,main }}(\mathcal{X}, L, \beta, \boldsymbol{x})$ has a virtual fundamental cycle of dimension $n$. Then we define

$$
n_{1, l, \beta}^{\mathcal{X}}\left([\mathrm{pt}]_{L} ; \mathbf{1}_{\nu_{1}}, \ldots, \mathbf{1}_{\nu_{l}}\right):=\operatorname{ev}_{0 *}\left(\left[\mathcal{M}_{1, l}^{\mathrm{op}, \text { main }}(\mathcal{X}, L, \beta, \boldsymbol{x})\right]^{\mathrm{vir}}\right) \in H_{n}(L ; \mathbb{Q}) \cong \mathbb{Q},
$$

where $\mathrm{ev}_{0}: \mathcal{M}_{1, l}^{\text {op,main }}(\mathcal{X}, L, \beta, \boldsymbol{x}) \rightarrow L$ is the evaluation map at the boundary marked point, $[\mathrm{pt}]_{L} \in H^{n}(L ; \mathbb{Q})$ is the point class of $L$, and $\mathbf{1}_{\nu_{j}} \in H^{0}\left(\mathcal{X}_{\nu_{j}} ; \mathbb{Q}\right) \subset H_{\mathrm{CR}}^{2 \text { age }\left(\nu_{j}\right)}(\mathcal{X} ; \mathbb{Q})$ is the fundamental class of the twisted sector $\mathcal{X}_{\nu_{j}}$.

Remark 2.7. The Kuranishi structures in this paper are the same as those defined in [14, 15], incorporating the works $[6,7]$ for the interior orbifold marked points. This has been explained in [9, Section 10]. We also refer the readers to [13, Appendix] and [16] for the detailed construction, and to [23] (and its forthcoming sequels) for a different approach.

The moduli spaces considered here are in fact much simpler than those in $[14,15]$ (and [13]) because we only need to consider stable disks with just one disk component which is minimal, and hence disk bubbling does not occur. In particular, we do not have codimension-one boundary components, and hence the above definition is independent of choices of Kuranishi perturbations.

For a basic (orbi-)disk with at most one interior orbifold marked point, the corresponding moduli space $\mathcal{M}_{1,0}^{\text {op,main }}\left(\mathcal{X}, L, \beta_{i}\right)\left(\operatorname{or} \mathcal{M}_{1,1}^{\text {op,main }}\left(\mathcal{X}, L, \beta_{\nu}, \nu\right)\right.$ when $\beta_{\nu}$ is a basic orbi-disk class) is regular and can be identified with $L$. Thus the associated invariants are evaluated as follows [9]:

1. For $\nu \in \mathrm{Box}^{\prime}$, we have $n_{1,1, \beta_{\nu}}^{\mathcal{X}}\left([\mathrm{pt}]_{L} ; \mathbf{1}_{\nu}\right)=1$.

2. For $i \in\{0, \ldots, m-1\}$, we have $n_{1,0, \beta_{i}}^{\mathcal{X}}\left([\mathrm{pt}]_{L}\right)=1$.

When there are more interior orbifold marked points or when the disk class is not basic, the corresponding moduli space is in general non-regular and virtual theory is involved in the definition, making the invariant much more difficult to compute.

\section{Geometric constructions}

Let $\beta \in \pi_{2}(\mathcal{X}, L)$ be a disk class with $\mu_{\mathrm{CW}}(\beta)=2$. By the discussion in Section 2.2, we can write

$$
\beta=\beta_{\mathbf{d}}+\alpha
$$

with $\alpha \in H_{2}(\mathcal{X}, \mathbb{Z}), c_{1}(\mathcal{X}) \cdot \alpha=0$ and either $\beta_{\mathbf{d}} \in\left\{\beta_{0}, \ldots, \beta_{m-1}\right\}$ or $\beta_{\mathbf{d}} \in \operatorname{Box}^{\prime}(\mathcal{X})$ is of age 1 . Denote by $\boldsymbol{b}_{d} \in N$ the element corresponding to $\beta_{\mathbf{d}}$.

Recall that the fan polytope $\mathcal{P} \subset N_{\mathbb{R}}$ is the convex hull of the vectors $\boldsymbol{b}_{0}, \ldots, \boldsymbol{b}_{m-1}$. Note that $\boldsymbol{b}_{d} \in \mathcal{P}$. Denote by $F\left(\boldsymbol{b}_{d}\right)$ the minimal face of the fan polytope $\mathcal{P}$ that contains the vector $\boldsymbol{b}_{d}$. Let $F$ be a facet of $\mathcal{P}$ that contains $F\left(\boldsymbol{b}_{d}\right)$. Let $\Sigma_{\beta_{\mathbf{d}}} \subset \Sigma$ be the minimal convex subfan containing all $\left\{\boldsymbol{b}_{0}, \ldots, \boldsymbol{b}_{m-1}\right\} \cap F$. The vectors

$$
\left\{\boldsymbol{b}_{0}, \ldots, \boldsymbol{b}_{m^{\prime}-1}\right\} \cap \sum_{\boldsymbol{b}_{j} \in\left\{\boldsymbol{b}_{0}, \ldots, \boldsymbol{b}_{m-1}\right\} \cap F} \mathbb{Q}_{\geq 0} \boldsymbol{b}_{j}
$$

\footnotetext{
${ }^{7}$ In the general case one may restrict to torus-equivariant perturbations, as did in $[14,15,17]$.
} 
determine a fan map $\mathbb{Z}^{p} \rightarrow N$ (where $p$ is the number of vectors above). Let

$$
\mathcal{X}_{\beta_{\mathbf{d}}} \subset \mathcal{X}
$$

be the associated toric suborbifold (of the same dimension $n$ ).

Lemma 3.1. $\mathcal{X}_{\beta_{\mathbf{d}}}$ is a toric Calabi-Yau orbifold.

Proof. All the generators in (3.1) lie in the hyperplane containing $F$. Since $\mathcal{X}$ is Gorenstein, this hyperplane has a defining equation $\nu=0$ for some primitive vector $\nu \in M$. Hence $\mathcal{X}_{\beta_{\mathbf{d}}}$ is toric Calabi-Yau.

Example 3.2. Consider $\mathbb{P}^{2} / \mathbb{Z}_{3}$, whose fan is shown in the left of Fig. 1. If $\beta_{\mathbf{d}}$ corresponds to the vector $(1,0)$ (which is marked as ' 113 ' in the figure), then $\Sigma_{\beta_{\mathbf{d}}}$ is the cone spanned by $v_{2}=(2,-1)$ and $v_{3}=(-1,2)$. If $\beta_{\mathbf{d}}$ corresponds to the vector $v_{3}$, then $\Sigma_{\beta_{\mathbf{d}}}$ can be taken to be the cone spanned by $v_{2}, v_{3}$, or the cone spanned by $v_{1}, v_{3}$. In both cases, the corresponding toric Calabi-Yau orbifold is $\mathbb{C}^{2} / \mathbb{Z}_{3}$.

Note that $\mathcal{X}_{\beta_{\mathbf{d}}}$ depends on the choice of the face $F$, not just $\beta_{\mathbf{d}}$. We use $\mathcal{X}_{\beta_{\mathbf{d}}}$ to compute open Gromov-Witten invariants of $\mathcal{X}$ in class $\beta=\beta_{\mathbf{d}}+\alpha$.

In what follows we show that $\mathcal{X}_{\beta_{\mathbf{d}}} \subset \mathcal{X}$ contains all stable orbi-disks of $\mathcal{X}$ of class $\beta$. First, we have the following analogue of [5, Proposition 5.6].

Lemma 3.3. Let $f: \mathcal{D} \cup \mathcal{C} \rightarrow \mathcal{X}$ be a stable orbi-disk map in the class $\beta=\beta_{\mathbf{d}}+\alpha$, where $\mathcal{D}$ is $a$ (possibly orbifold) disk and $\mathcal{C}$ is a (possibly orbifold) rational curve such that $f_{*}[\mathcal{D}]=\beta_{\mathbf{d}}$ and $f_{*}[\mathcal{C}]=\alpha$ with $c_{1}(\alpha)=0$. Then we have

$$
f(\mathcal{C}) \subset \bigcup_{\boldsymbol{b}_{j} \in F\left(\boldsymbol{b}_{d}\right)} D_{j}
$$

and $[f(\mathcal{C})] \cdot D_{j}=0$ whenever $\boldsymbol{b}_{j} \notin F\left(\boldsymbol{b}_{d}\right)$.

Proof. Since $c_{1}(\alpha)=0, f(\mathcal{C})$ should lie in toric divisors of $\mathcal{X}$. Recall that $\beta_{\mathbf{d}}$ achieves the minimal Maslov index 2, and hence there is no disc bubbling.

Suppose $\beta_{\mathbf{d}}$ is a smooth disk class. Then each sphere component $\mathcal{C}_{0}$ meeting the disk component $\mathcal{D}$ maps into the divisor $D_{\mathbf{d}}$ and it should have non-negative intersection with other toric divisors. By [18, Lemma 4.5] which easily extends to the simplicial setting, we have the desired statement for $f\left(\mathcal{C}_{0}\right)$.

If $\beta_{\mathbf{d}}$ is an orbi-disk class, then we can write the corresponding $\boldsymbol{b}_{d} \in N$ as $\boldsymbol{b}_{d}=\sum_{\boldsymbol{b}_{i} \in \sigma} c_{i} \boldsymbol{b}_{i}$, with $\sum_{i} c_{i}=1, c_{i} \in[0,1) \cap \mathbb{Q}$. For a sphere component $\mathcal{C}_{0}$ meeting the disk component $\mathcal{D}$, we have $f\left(\mathcal{C}_{0}\right) \subset \bigcup_{\boldsymbol{b}_{i} \in \sigma} D_{i}$ and each $\boldsymbol{b}_{i} \in \sigma$ satisfies $\boldsymbol{b}_{i} \in F\left(\boldsymbol{b}_{d}\right)$. Hence $f\left(\mathcal{C}_{0}\right) \subset \bigcup_{\boldsymbol{b}_{i} \in F\left(\boldsymbol{b}_{d}\right)} D_{i}$ and $f\left(\mathcal{C}_{0}\right) \cdot D_{j}=0$ for $\boldsymbol{b}_{j} \notin F\left(\boldsymbol{b}_{d}\right)$.

Let $\mathcal{C}_{1} \subset \mathcal{C}$ be a sphere component meeting $\mathcal{C}_{0}$, then we have $f\left(\mathcal{C}_{1}\right) \subset F\left(\boldsymbol{b}_{j}\right)$ for some $\boldsymbol{b}_{j} \in F\left(\boldsymbol{b}_{d}\right)$ by the intersection condition. Now, we can follow the proof of [5, Proposition 5.6] shows that $f\left(\mathcal{C}_{1}\right) \subset \bigcup_{\boldsymbol{b}_{i} \in F\left(\boldsymbol{b}_{d}\right)} D_{i}$. The result follows by repeating this argument for one sphere component at a time.

Partition $\left\{\boldsymbol{b}_{0}, \ldots, \boldsymbol{b}_{m-1}\right\} \cap F\left(\boldsymbol{b}_{d}\right)$ into the disjoint union of two subsets,

$$
\left\{\boldsymbol{b}_{0}, \ldots, \boldsymbol{b}_{m-1}\right\} \cap F\left(\boldsymbol{b}_{d}\right)=F\left(\boldsymbol{b}_{d}\right)^{c} \coprod F\left(\boldsymbol{b}_{d}\right)^{n c},
$$

where $\boldsymbol{b}_{i} \in F\left(\boldsymbol{b}_{d}\right)^{c}$ if $D_{i} \subset \mathcal{X}_{\beta_{\mathbf{d}}}$ and $\boldsymbol{b}_{i} \in F\left(\boldsymbol{b}_{d}\right)^{n c}$ if $D_{i} \not \subset \mathcal{X}_{\beta_{\mathbf{d}}}$. 
Lemma 3.4. Let $f: \mathcal{D} \cup \mathcal{C} \rightarrow \mathcal{X}$ be as in Lemma 3.3. Then we have $f(\mathcal{D} \cup \mathcal{C}) \subset \mathcal{X}_{\beta_{\mathbf{d}}}$.

Proof. Certainly $f(\mathcal{D}) \subset \mathcal{X}_{\beta_{\mathbf{d}}}$. We claim that

$$
f(\mathcal{C}) \subset \bigcup_{\boldsymbol{b}_{j} \in F\left(\boldsymbol{b}_{d}\right)^{c}} D_{j}
$$

from which the lemma follows.

To see (3.2), we write $\mathcal{C}=\mathcal{C}_{c} \cup \mathcal{C}_{n c}$ where $\mathcal{C}_{c}$ consists of components of $\mathcal{C}$ which lie in $\bigcup D_{j}$, and $\mathcal{C}_{n c}$ consists of the remaining components. Set $A:=f_{*}\left[\mathcal{C}_{c}\right]$ and $B:=f_{*}\left[\mathcal{C}_{n c}\right]$. $\boldsymbol{b}_{j} \in F\left(\boldsymbol{b}_{d}\right)^{c}$

Then $\alpha=A+B$. Since $-K_{\mathcal{X}}$ is nef and $-K_{\mathcal{X}} \cdot \alpha=0$, we have $-K_{\mathcal{X}} \cdot A=0=-K_{\mathcal{X}} \cdot B$. Write $B=\sum_{k} c_{k} B_{k}$ as an effective linear combination of the classes $B_{k}$ of irreducible 1-dimensional torus-invariant orbits in $\mathcal{X}$. Again because $-K_{\mathcal{X}}$ is nef, we have $-K_{\mathcal{X}} \cdot B_{k}=0$ for all $k$. Each $B_{k}$ corresponds to an $(n-1)$-dimensional cone $\sigma_{k} \in \Sigma$. In the expression $B=\sum_{k} c_{k} B_{k}$, there is at least one (non-zero) $B_{k}$ which is not contained in $\bigcup_{\boldsymbol{b}_{j} \in F\left(\boldsymbol{b}_{d}\right)^{c}} D_{j}$. As a consequence, either $\sigma_{k}$ contains a ray $\mathbb{R}_{\geq 0} \boldsymbol{b}_{j}$ with $\boldsymbol{b}_{j} \notin F\left(\boldsymbol{b}_{d}\right)$, or there exists a $\boldsymbol{b}_{j} \notin F\left(\boldsymbol{b}_{d}\right)$ such that $\sigma_{k}$ and $\boldsymbol{b}_{j}$ span an $n$-dimensional cone in $\Sigma$.

Since $B_{k}$ is not contained in $\bigcup_{\boldsymbol{b}_{j} \in F\left(\boldsymbol{b}_{d}\right)^{c}} D_{j}$, we see that if $\boldsymbol{b}_{i} \in F\left(\boldsymbol{b}_{d}\right)^{c}$ then $\boldsymbol{b}_{i} \notin \sigma_{k}$. Also, $D \cdot B_{k} \geq 0$ for every toric prime divisor $D \subset \mathcal{X}$ not corresponding to a ray in $\sigma_{k}$.

By [18, Lemma 4.5] (which easily extends to the simplical setting), we have $D \cdot B_{k}=0$ for every toric prime divisor $D \subset \mathcal{X}$ corresponding to an element in $\left\{\boldsymbol{b}_{1}, \ldots, \boldsymbol{b}_{m}\right\} \backslash F\left(\sigma_{k}\right)$, where $F\left(\sigma_{k}\right) \subset \mathcal{P}$ is the minimal face of $\mathcal{P}$ containing rays in $\sigma_{k}$. Since the divisors $D \subset \mathcal{X}$ corresponding to $\left\{\boldsymbol{b}_{1}, \ldots, \boldsymbol{b}_{m}\right\} \backslash F\left(\sigma_{k}\right)$ span $H^{2}(\mathcal{X})$, we have $B_{k}=0$, a contradiction.

Let $\boldsymbol{x}=\left(\mathcal{X}_{\nu_{1}}, \ldots, \mathcal{X}_{\nu_{l}}\right)$ be an $l$-tuple of twisted sectors of $\mathcal{X}_{\beta_{\mathbf{d}}}$. Then Lemma 3.4 implies that the natural inclusion $\mathcal{M}_{1, l}^{\text {op,main }}\left(\mathcal{X}_{\beta_{\mathbf{d}}}, L, \beta, \boldsymbol{x}\right) \hookrightarrow \mathcal{M}_{1, l}^{\text {op,main }}(\mathcal{X}, L, \beta, \boldsymbol{x})$ is a bijection. Since $\mathcal{X}_{\beta_{\mathbf{d}}} \subset \mathcal{X}$ is open, the local deformations and obstructions of stable discs in $\mathcal{X}_{\beta_{\mathbf{d}}}$ and their inclusion in $\mathcal{X}$ are isomorphic. It follows that

Proposition 3.5. The moduli spaces $\mathcal{M}_{1, l}^{\text {op,main }}(\mathcal{X}, L, \beta, \boldsymbol{x})$ of disks in $\mathcal{X}$ is isomorphic as Kuranishi spaces to the moduli spaces $\mathcal{M}_{1, l}^{\text {op,main }}\left(\mathcal{X}_{\beta_{\mathbf{d}}}, L, \beta, \boldsymbol{x}\right)$ of disks in $\mathcal{X}_{\beta_{\mathbf{d}}}$. Consequently

$$
n_{1, l, \beta}^{\mathcal{X}}\left([\mathrm{pt}]_{L} ; \mathbf{1}_{\nu_{1}}, \ldots, \mathbf{1}_{\nu_{l}}\right)=n_{1, l, \beta}^{\mathcal{X}_{\beta_{\mathrm{d}}}}\left([\mathrm{pt}]_{L} ; \mathbf{1}_{\nu_{1}}, \ldots, \mathbf{1}_{\nu_{l}}\right) .
$$

Since $\mathcal{X}_{\beta_{\mathbf{d}}}$ is a toric Calabi-Yau orbifold, the open Gromov-Witten invariants $n_{1, l, \beta} \mathcal{X}_{\beta_{\mathbf{d}}}\left([\mathrm{pt}]_{L}\right.$; $\mathbf{1}_{\nu_{1}}, \ldots, \mathbf{1}_{\nu_{l}}$ ) have been computed in [4]. By Proposition 3.5, this gives open Gromov-Witten invariants of $\mathcal{X}$. Explicitly they are given as follows.

Using the toric data of $\mathcal{X}_{\beta_{\mathbf{d}}}$, we define

$$
\begin{aligned}
& \Omega_{j}^{\mathcal{X}_{\beta_{\mathbf{d}}}}:=\left\{d \in \mathbb{K}_{\mathrm{eff}} \mid \nu(d)=0,\left\langle D_{j}, d\right\rangle \in \mathbb{Z}_{<0}\right. \text { and } \\
& \left.\left\langle D_{i}, d\right\rangle \in \mathbb{Z}_{\geq 0} \forall i \neq j\right\}, \quad j=0,1, \ldots, m-1, \\
& \Omega_{j}^{\mathcal{X}_{\beta_{\mathbf{d}}}}:=\left\{d \in \mathbb{K}_{\mathrm{eff}} \mid \nu(d)=\boldsymbol{b}_{j} \text { and }\left\langle D_{i}, d\right\rangle \notin \mathbb{Z}_{<0} \forall i\right\}, \quad j=m, m+1, \ldots, m^{\prime}-1, \\
& A_{j}^{\mathcal{X}_{\beta_{\mathbf{d}}}}(y):=\sum_{d \in \Omega_{j}^{\mathcal{X}_{\beta_{\mathbf{d}}}}} y^{d} \frac{(-1)^{-\left\langle D_{j}, d\right\rangle-1}\left(-\left\langle D_{j}, d\right\rangle-1\right) !}{\prod_{i \neq j}\left\langle D_{i}, d\right\rangle !}, \quad j=0,1, \ldots, m-1, \\
& A_{j}^{\mathcal{X}_{\beta_{\mathbf{d}}}}(y):=\sum_{d \in \Omega_{j}^{\mathcal{X}_{\beta_{\mathbf{d}}}}} y^{d} \prod_{i=0}^{m^{\prime}-1} \frac{\prod_{k=\left\lceil\left\langle D_{i}, d\right\rangle\right\rceil}^{\infty}\left(\left\langle D_{i}, d\right\rangle-k\right)}{\prod_{k=0}^{\infty}\left(\left\langle D_{i}, d\right\rangle-k\right)}, \quad j=m, m+1, \ldots, m^{\prime}-1,
\end{aligned}
$$




$$
\begin{aligned}
& \log q_{a}=\log y_{a}+\sum_{j=0}^{m-1} Q_{j a} A_{j}^{\mathcal{X}_{\beta_{\mathbf{d}}}}(y), \quad a=1, \ldots, r^{\prime}, \\
& \tau_{\boldsymbol{b}_{j}}=A_{j}^{\mathcal{X}_{\beta_{\mathbf{d}}}}(y), \quad j=m, \ldots, m^{\prime}-1,
\end{aligned}
$$

Theorem 3.6. If $\beta_{\mathbf{d}}=\beta_{i_{0}}$ is a basic smooth disk class corresponding to the ray generated by $\boldsymbol{b}_{i_{0}}$ for some $i_{0} \in\{0,1, \ldots, m-1\}$, then we have

$$
\begin{aligned}
& \sum_{\alpha \in H_{2}^{\text {eff }}(\mathcal{X})} \sum_{l \geq 0} \sum_{\nu_{1}, \ldots, \nu_{l} \in \operatorname{Box}^{\prime}\left(\Sigma_{\beta_{\mathbf{d}}}\right)} \frac{\prod_{i=1}^{l} \tau_{\nu_{i}}}{l !} n_{1, l, \beta_{i_{0}}+\alpha}^{\mathcal{X}}\left([\mathrm{pt}]_{L} ; \prod_{i=1}^{l} \mathbf{1}_{\nu_{i}}\right) q^{\alpha} \\
& =\exp \left(-A_{i_{0}}^{\mathcal{X}_{\beta_{\mathbf{d}}}}(y(q, \tau))\right)
\end{aligned}
$$

via the inverse $y=y(q, \tau)$ of the toric mirror map (3.3).

If $\beta_{\mathbf{d}}=\beta_{\nu_{0}}$ is a basic orbi-disk class corresponding to $\nu_{j_{0}} \in \operatorname{Box}^{\prime}(\Sigma)^{\text {age }=1}$ for some $j_{0} \in$ $\left\{m, m+1, \ldots, m^{\prime}-1\right\}$, then we have

$$
\begin{gathered}
\sum_{\alpha \in H_{2}^{\text {eff }}(\mathcal{X})} \sum_{l \geq 0} \sum_{\nu_{1}, \ldots, \nu_{l} \in \operatorname{Box}^{\prime}\left(\Sigma_{\beta_{\mathbf{d}}}\right)} \frac{\prod_{i=1}^{l} \tau_{\nu_{i}}}{l !} n_{1, l, \beta_{\nu_{j}}+\alpha}^{\mathcal{X}}\left([\mathrm{pt}]_{L} ; \prod_{i=1}^{l} \mathbf{1}_{\nu_{i}}\right) q^{\alpha} \\
=y^{D_{j_{0}}^{\vee} \exp }\left(-\sum_{i \notin I_{j_{0}}} c_{j_{0} i} A_{i}^{\mathcal{X}_{\beta_{\mathbf{d}}}}(y(q, \tau))\right),
\end{gathered}
$$

via the inverse $y=y(q, \tau)$ of the toric mirror map (3.3), where $D_{j_{0}}^{\vee} \in \mathbb{K}_{\mathrm{eff}}$ is the class defined in (2.4), $I_{j_{0}} \in \mathcal{A}$ is the anticone of the minimal cone containing $\boldsymbol{b}_{j_{0}}=\nu_{j_{0}}$ and $c_{j_{0} i} \in \mathbb{Q} \cap[0,1)$ are rational numbers such that $\boldsymbol{b}_{j_{0}}=\sum_{i \notin I_{j_{0}}} c_{j_{0} i} \boldsymbol{b}_{i}$.

Proof. By Proposition 3.5, $n_{1, l, \beta}^{\mathcal{X}}\left([\mathrm{pt}]_{L} ; \mathbf{1}_{\nu_{1}}, \ldots, \mathbf{1}_{\nu_{l}}\right)=n_{1, l, \beta}^{\mathcal{X}_{\beta_{\mathbf{d}}}}\left([\mathrm{pt}]_{L} ; \mathbf{1}_{\nu_{1}}, \ldots, \mathbf{1}_{\nu_{l}}\right)$, and so the 1.h.s. of (3.4) is equal to

$$
\sum_{\alpha \in H_{2}^{\text {eff }}\left(\mathcal{X}_{\beta_{\mathbf{d}}}\right)} \sum_{l \geq 0} \sum_{\nu_{1}, \ldots, \nu_{l} \in \operatorname{Box}^{\prime}\left(\Sigma_{\beta_{\mathbf{d}}}\right)} \frac{\prod_{i=1}^{l} \tau_{\nu_{i}}}{l !} n_{1, l, \beta_{i_{0}}+\alpha}^{\mathcal{X}_{\beta_{\mathbf{d}}=1}}\left([\mathrm{pt}]_{L} ; \prod_{i=1}^{l} \mathbf{1}_{\nu_{i}}\right) q^{\alpha},
$$

which in turn is equal to $\exp \left(-A_{i_{0}}^{\mathcal{X}_{\beta_{\mathbf{d}}}}(y(q, \tau))\right)$ by [4, Theorem 1.4]. The deduction for (3.5) is similar.

To combine all the invariants into a single expression, one defines the orbi-disc potential

$$
W=\sum_{\beta_{\mathbf{d}}} \sum_{\alpha \in H_{2}^{\text {eff }}(\mathcal{X})} \sum_{l \geq 0} \sum_{\nu_{1}, \ldots, \nu_{l} \in \operatorname{Box}^{\prime}\left(\Sigma_{\beta_{\mathbf{d}}}\right)} \frac{\prod_{i=1}^{l} \tau_{\nu_{i}}}{l !} q^{\alpha} n_{1, l, \beta_{\mathbf{d}}+\alpha}^{\mathcal{X}}\left([\mathrm{pt}]_{L} ; \prod_{i=1}^{l} \mathbf{1}_{\nu_{i}}\right) Z^{\beta_{\mathbf{d}}}
$$

where $\beta_{\mathbf{d}}$ runs over all the basic smooth or orbi-disc classes, and $Z^{\beta_{\mathbf{d}}}$ are monomials associated to $\beta_{\mathbf{d}}$. See [5, Definition 19] for more detail. The above theorem gives an explicit expression of $W$ via the mirror map. 
Example 3.7. $\mathbb{P}^{2} / \mathbb{Z}_{3}$ is a Gorenstein Fano toric orbifold. Its fan and polytope pictures are shown in Fig. 1. It has three toric divisors $D_{1}, D_{2}, D_{3}$ corresponding to the rays generated by $v_{1}=(-1,-1), v_{2}=(2,-1), v_{3}=(-1,2)$. By pairing with the dual vectors $(1,0)$ and $(0,1)$, the linear equivalence relations are $2 D_{2}-D_{3}-D_{1} \sim 0$ and $2 D_{3}-D_{2}-D_{1} \sim 0$, and so $D_{1} \sim D_{2} \sim D_{3}$. It has three orbifold points corresponding to the three vertices in the polytope picture. Locally it is $\mathbb{C}^{2} / \mathbb{Z}_{3}$ around each orbifold point.
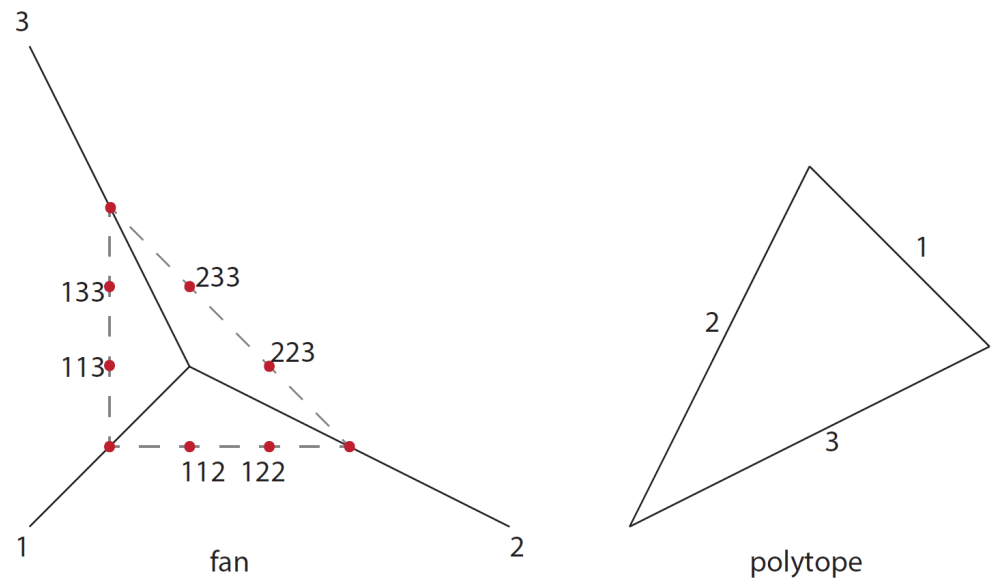

Figure 1. The fan and polytope picture for $\mathbb{P}^{2} / \mathbb{Z}_{3}$.

Fix a Lagrangian torus fiber. $\mathbb{P}^{2} / \mathbb{Z}_{3}$ has nine basic orbi-disk classes corresponding to the nine lattice points on the boundary of the fan polytope. Three of them are smooth disk classes and denote them by $\beta_{1}, \beta_{2}, \beta_{3}$. The basic orbi-disk classes corresponding to the two lattice points $\left(2 v_{1}+v_{2}\right) / 3$ and $\left(v_{1}+2 v_{2}\right) / 3$ are denoted by $\beta_{112}$ and $\beta_{122}$, which pass through the twisted sectors $\nu_{112}$ and $\nu_{122}$ respectively. Then $2 \beta_{1}+\beta_{2}-3 \beta_{112}$ (or $2 \beta_{2}+\beta_{1}-3 \beta_{122}$ ) is the class of a constant orbi-sphere passing through the twisted sector $\nu_{112}$ (or $\nu_{122}$ resp.). In particular the area of $\beta_{112}$ equals to $\left(2 \beta_{1}+\beta_{2}\right) / 3$. Other basic orbi-disk classes have similar notations.

Theorem 3.6 provides a formula for the open GW invariants $n_{1, l, \beta_{112}}^{\mathcal{X}}\left([\mathrm{pt}]_{L} ; \prod_{i=1}^{l} \mathbf{1}_{\nu_{i}}\right)$ where $\nu_{i}$ is either $\nu_{112}$ or $\nu_{122}$ for each $i$. To write down the invariants more systematically, we consider the open GW potential as follows.

Let $q$ be the Kähler parameter of the smooth sphere class $\beta_{1}+\beta_{2}+\beta_{3} \in H_{2}\left(\mathbb{P}^{2} / \mathbb{Z}_{3}\right)$. The basic orbi-disk classes correspond to monomials in the disk potential $q^{\beta} z^{\partial \beta}$, where $q^{\beta_{1}}=q^{\beta_{2}}=$ $q^{\beta_{112}}=q^{\beta_{122}}=1, q^{\beta_{3}}=q^{\beta_{1}+\beta_{2}+\beta_{3}}=q, q^{\beta_{223}}=q^{\left(2 \beta_{2}+\beta_{3}\right) / 3}=q^{\beta_{3} / 3}=q^{1 / 3}$, and similar for other basic orbi-disk classes. The Kähler parameters corresponding to the twisted sectors $\nu_{112}, \nu_{122}$ are denoted as $\tau_{112}, \tau_{122}$ (and similar for other twisted sectors).

By [4, Example 1, Section 6.5], the open GW potential for $\mathbb{C}^{2} / \mathbb{Z}_{3}$ is given by

$$
w\left(z-\kappa_{0}\left(\tau_{112}, \tau_{122}\right)\right)\left(z-\kappa_{1}\left(\tau_{112}, \tau_{122}\right)\right)\left(z-\kappa_{2}\left(\tau_{112}, \tau_{122}\right)\right)
$$

where

$$
\kappa_{k}\left(\tau_{1}, \tau_{2}\right)=\zeta^{2 k+1} \prod_{r=1}^{2} \exp \left(\frac{1}{3} \zeta^{(2 k+1) r} \tau_{r}\right), \quad \zeta:=\exp (\pi \sqrt{-1} / 3) .
$$

By Proposition 3.5 , the disk invariants of $\mathbb{P}^{2} / \mathbb{Z}_{3}$ equal to those of $\mathbb{C}^{2} / \mathbb{Z}_{3}$. Thus the open GW potential of $\mathbb{P}^{2} / \mathbb{Z}_{3}$ is given by

$$
W=z^{-1} w^{-1}\left(z-\kappa_{0}\left(\tau_{112}, \tau_{122}\right)\right)\left(z-\kappa_{1}\left(\tau_{112}, \tau_{122}\right)\right)\left(z-\kappa_{2}\left(\tau_{112}, \tau_{122}\right)\right)
$$




$$
\begin{aligned}
& +z^{-1} w^{-1}\left(q^{1 / 3} w-\kappa_{0}\left(\tau_{113}, \tau_{133}\right)\right)\left(q^{1 / 3} w-\kappa_{1}\left(\tau_{113}, \tau_{133}\right)\right)\left(q^{1 / 3} w-\kappa_{2}\left(\tau_{113}, \tau_{133}\right)\right) \\
& +z^{2} w^{-1}\left(q^{1 / 3} z^{-1} w-\kappa_{0}\left(\tau_{223}, \tau_{233}\right)\right)\left(q^{1 / 3} z^{-1} w-\kappa_{1}\left(\tau_{223}, \tau_{233}\right)\right) \\
& \times\left(q^{1 / 3} z^{-1} w-\kappa_{2}\left(\tau_{223}, \tau_{233}\right)\right)-z^{-1} w^{-1}-z^{2} w^{-1}-q z^{-1} w^{2}
\end{aligned}
$$

Then the generating functions of open orbifold GW for $\beta_{112}$ and $\beta_{122}$ are given by the coefficients of $w^{-1}$ and $z w^{-1}$ in $W$ respectively. The first few terms are given by the following table.

\begin{tabular}{|c|c|c|c|c|c|c|c|}
\hline$n_{(a, b)}$ & $a=0$ & $a=1$ & $a=2$ & $a=3$ & $a=4$ & $a=5$ & $a=6$ \\
\hline$b=0$ & 0 & 1 & 0 & 0 & $1 / 648$ & 0 & 0 \\
\hline$b=1$ & 0 & 0 & $-1 / 18$ & 0 & 0 & $-1 / 29160$ & 0 \\
\hline$b=2$ & $1 / 6$ & 0 & 0 & $1 / 972$ & 0 & 0 & $1 / 3149280$ \\
\hline$b=3$ & 0 & $-1 / 162$ & 0 & 0 & $-1 / 104976$ & 0 & 0 \\
\hline$b=4$ & 0 & 0 & $1 / 11664$ & 0 & 0 & $1 / 18895680$ & 0 \\
\hline$b=5$ & $-1 / 9720$ & 0 & 0 & $-1 / 1574640$ & 0 & 0 & $-1 / 5101833600$ \\
\hline$b=6$ & 0 & $1 / 524880$ & 0 & 0 & $1 / 340122240$ & 0 & 0 \\
\hline
\end{tabular}

In the above table,

$$
n_{(a, b)}=n_{1, a+b, \beta_{112}}\left([\mathrm{pt}]_{L} ; \mathbf{1}_{\nu_{112}}^{\otimes a}, \mathbf{1}_{\nu_{122}}^{\otimes b}\right)=n_{1, a+b, \beta_{122}}\left([\mathrm{pt}]_{L} ; \mathbf{1}_{\nu_{112}}^{\otimes b}, \mathbf{1}_{\nu_{122}}^{\otimes a}\right) .
$$

We observe that all invariants satisfy 'reciprocal integrality', namely their reciprocals are integers. Moreover, all these integers are divisible by $6 . n_{(k, k)}=0$. Furthermore, the sign is alternating with respect to $b$.

\section{Acknowledgments}

K. Chan was supported by a Hong Kong RGC grant CUHK14314516 and direct grants from CUHK. C.-H. Cho was supported by the NRF grant funded by the Korea government(MSIT) (No. 2017R1A22B4009488). S.-C. Lau was partially supported by the Simons collaboration grant \#580648. N.C. Leung was supported by Hong Kong RGC grants CUHK14302215 \& CUHK14303516 and direct grants from CUHK. H.-H. Tseng was supported in part by NSF grant DMS-1506551.

\section{References}

[1] Auroux D., Mirror symmetry and T-duality in the complement of an anticanonical divisor, J. Gökova Geom. Topol. 1 (2007), 51-91, arXiv:0706.3207.

[2] Borisov L.A., Chen L., Smith G.G., The orbifold Chow ring of toric Deligne-Mumford stacks, J. Amer. Math. Soc. 18 (2005), 193-215, arXiv:math.AG/0309229.

[3] Chan K., Cho C.-H., Lau S.-C., Tseng H.-H., Lagrangian Floer superpotentials and crepant resolutions for toric orbifolds, Comm. Math. Phys. 328 (2014), 83-130, arXiv:1208.5282.

[4] Chan K., Cho C.-H., Lau S.-C., Tseng H.-H., Gross fibrations, SYZ mirror symmetry, and open Gromov-Witten invariants for toric Calabi-Yau orbifolds, J. Differential Geom. 103 (2016), 207-288, arXiv:1306.0437.

[5] Chan K., Lau S.-C., Leung N.C., Tseng H.-H., Open Gromov-Witten invariants, mirror maps, and Seidel representations for toric manifolds, Duke Math. J. 166 (2017), 1405-1462, arXiv:1209.6119.

[6] Chen W., Ruan Y., Orbifold Gromov-Witten theory, in Orbifolds in Mathematics and Physics (Madison, WI, 2001), Contemp. Math., Vol. 310, Amer. Math. Soc., Providence, RI, 2002, 25-85, arXiv:math.AG/0103156.

[7] Chen W., Ruan Y., A new cohomology theory of orbifold, Comm. Math. Phys. 248 (2004), 1-31, arXiv:math.AG/0004129.

[8] Cho C.-H., Oh Y.-G., Floer cohomology and disc instantons of Lagrangian torus fibers in Fano toric manifolds, Asian J. Math. 10 (2006), 773-814, arXiv:math.SG/0308225. 
[9] Cho C.-H., Poddar M., Holomorphic orbi-discs and Lagrangian Floer cohomology of symplectic toric orbifolds, J. Differential Geom. 98 (2014), 21-116, arXiv:1206.3994.

[10] Cho C.-H., Shin H.-S., Chern-Weil Maslov index and its orbifold analogue, Asian J. Math. 20 (2016), 1-19, arXiv:1202.0556.

[11] Coates T., Corti A., Iritani H., Tseng H.-H., A mirror theorem for toric stacks, Compos. Math. 151 (2015), 1878-1912, arXiv:1310.4163.

[12] Cox D.A., Little J.B., Schenck H.K., Toric varieties, Graduate Studies in Mathematics, Vol. 124, Amer. Math. Soc., Providence, RI, 2011.

[13] Fukaya K., Oh Y.-G., Ohta H., Ono K., Lagrangian intersection Floer theory: anomaly and obstruction, AMS/IP Studies in Advanced Mathematics, Vol. 46, Amer. Math. Soc., Providence, RI, 2009.

[14] Fukaya K., Oh Y.-G., Ohta H., Ono K., Lagrangian Floer theory on compact toric manifolds. I, Duke Math. J. 151 (2010), 23-174, arXiv:0802.1703.

[15] Fukaya K., Oh Y.-G., Ohta H., Ono K., Lagrangian Floer theory on compact toric manifolds II: bulk deformations, Selecta Math. (N.S.) 17 (2011), 609-711, arXiv:0810.5654.

[16] Fukaya K., Oh Y.-G., Ohta H., Ono K., Technical details on Kuranishi structure and virtual fundamental chain, arXiv:1209.4410.

[17] Fukaya K., Oh Y.-G., Ohta H., Ono K., Lagrangian Floer theory and mirror symmetry on compact toric manifolds, Astérisque 376 (2016), vi+340 pages, arXiv:1009.1648.

[18] González E., Iritani H., Seidel elements and mirror transformations, Selecta Math. (N.S.) 18 (2012), 557590, arXiv:1103.4171.

[19] Gross M., Siebert B., From real affine geometry to complex geometry, Ann. of Math. 174 (2011), 1301-1428, arXiv:math.AG/0703822.

[20] Hausel T., Sturmfels B., Toric hyperKähler varieties, Doc. Math. 7 (2002), 495-534, arXiv:math.AG/0203096.

[21] Iritani H., An integral structure in quantum cohomology and mirror symmetry for toric orbifolds, $A d v$. Math. 222 (2009), 1016-1079, arXiv:0903.1463.

[22] Jiang Y., The orbifold cohomology ring of simplicial toric stack bundles, Illinois J. Math. 52 (2008), 493-514, arXiv:math.AG/0504563.

[23] McDuff D., Wehrheim K., Smooth Kuranishi atlases with isotropy, Geom. Topol. 21 (2017), 2725-2809, arXiv:1508.01556.

[24] Ruddat H., Siebert B., Period integrals from wall structures via tropical cycles, canonical coordinates in mirror symmetry and analyticity of toric degenerations, Publ. Math. Inst. Hautes Études Sci., to appear, arXiv:1907.03794.

[25] Strominger A., Yau S.-T., Zaslow E., Mirror symmetry is T-duality, Nuclear Phys. B 479 (1996), 243-259, arXiv:hep-th/9606040. 